\title{
Broadband and Temperature Tolerant Silicon Nitride Liquid Controlled Waveguide Coupler
}

\author{
Herbert D’heer, Kumar Saurav, Cristina Lerma Arce, Salvatore Tuccio, Stéphane Clemmen, Silvia Lenci, \\ Andim Stassen, Jan Watté, and Dries Van Thourhout
}

\begin{abstract}
A broadband and temperature tolerant liquidcontrolled adiabatic waveguide coupler (LCC) is realized. The LCC with silicon nitride ( $\mathrm{SiN}$ ) waveguides is designed to be compatible with liquids that can be actuated by an electrowetting-ondielectric (EWOD) system. This proof-of-principle demonstration with manual actuation paves the way towards the realization of non-volatile optical switch systems. A $630 \mu \mathrm{m}$ long LCC configured as a $1 \times 2$ switch has a measured insertion loss less than $1.5 \mathrm{~dB}$ and a crosstalk less than $-14 \mathrm{~dB}$ for both bar and cross state over the telecommunication wavelength range $1260 \mathrm{~nm}$ to $1630 \mathrm{~nm}$. Furthermore, the LCC is tolerant to variations in temperature. The measured excess insertion loss over the temperature range $21^{\circ} \mathrm{C}$ to $73^{\circ} \mathrm{C}$ is less than $0.3 \mathrm{~dB}$ for bar and cross state, over the same wavelength range.
\end{abstract}

Index Terms-Couplers, integrated optics, liquids, optical fiber communication, optical switches, silicon nitride, silicon photonics.

\section{INTRODUCTION}

O PTICAL switches are an essential part of reconfigurable fiber networks. Highly desirable features are low optical loss, wide operating spectral window, and low power consumption both for switching and during steady state operation. Optical micro-electromechanical systems (MEMS) are currently the standard in this context. However, they consume energy in a steady state operation. Non-volatile switches have been realized by using, for example, buckling waveguides [1], or latching MEMS [2] and liquids [3]. Other qualities desired for a switch are: a wideband operation covering the telecom $\mathrm{O}, \mathrm{S}, \mathrm{C}$ and $\mathrm{L}$ bands, a compact size and the possibility to create switches with many ports. Switches based on waveguide couplers that are broadband and compact have been reported in literature, but they are volatile and do not cover the full telecommunication wavelength range with good performance [4]-[6].

Silicon photonics switches with waveguide couplers can be designed such that they do not consume energy in a steady state and have the additional advantages of being very compact and potentially low cost. Previously we demonstrated the design and fabrication of liquid controlled adiabatic couplers (LCC) with silicon waveguides [3], [7]. Compared to earlier

This work was supported in part by the EU FP7 SwIFT Project with reference 619643 .

H. D'heer, S. Clemmen, and D. Van Thourhout are with the Photonics Research Group, Department of Information Technology (INTEC), Ghent University - imec, 9052 Gent, Belgium.

K. Saurav, C. Lerma Arce, S. Tuccio, and J. Watté are with CommScope, 3010 Kessel-Lo, Belgium

S. Lenci and A. Stassen are with imec, Kapeldreef 75, 3001 Leuven, Belgium demonstrated liquid controlled optical switches [8]-[10] these have the advantage that they can be directly integrated with a standardised waveguide platform, readily available from multiple foundries. However, the wavelength range of operation was restricted to about $100 \mathrm{~nm}$ which hinders the application in telecommunication fiber networks where broadband operation over the wavelength range $1260 \mathrm{~nm}$ to $1630 \mathrm{~nm}$ is desired. The length of the LCCs in [3] is up to $1.4 \mathrm{~mm}$. In the present paper we show that LCCs with silicon nitride ( $\mathrm{SiN}$ ) waveguides can be short, with a length of $630 \mu \mathrm{m}$, while they can operate over a broad wavelength range from $1260 \mathrm{~nm}$ to $1630 \mathrm{~nm}$ with good performance. We also show that the fabricated LCC is tolerant to temperature variations. The temperature can vary from at least $21^{\circ} \mathrm{C}$ to $73^{\circ} \mathrm{C}$ with only a small influence on its performance therefore avoiding the need of an active temperature control circuit. This allows the switch to operate in an uncontrolled environment with zero energy consumption after switching.

The performance of the previously demonstrated siliconbased LCCs is restricted by the large confinement of the optical mode inside the waveguides. A larger overlap of the optical mode with the liquid, and thus better performance, can be realized by reducing the waveguide thickness down to about $100 \mathrm{~nm}$ as proposed in [3]. Reducing the waveguide thickness, however, requires non-standard silicon-on-insulator (SOI) wafers with a sufficiently thick buried oxide.

Increasing the overlap of the optical mode with the liquid can also be realized by a waveguide core with a reduced refractive index, such as SiN. This material can be deposited by a chemical vapor deposition process which allows a higher flexibility in waveguide thickness than SOI technology and also allows a thick buried oxide. The refractive index of SiN is still sufficiently large to achieve a fairly large refractive index variation as a function of waveguide width, which is also required to realize short LCCs with good performance.

In practical applications it is desired that the movement of the liquids above the LCCs on a chip is done by a compact fluidic system. An electrowetting-on-dielectric (EWOD) system, such as described in [11], is a good candidate for this. It enables the movement of small optically transparent liquid drops above the optical chip and at the same time, when properly designed, it is non-volatile. This means that the liquid drops will not move when the electrical power is switched off, even when the system is mechanically shaked, and therefore the optical switch will also remain in a stable state. In this paper the liquids are manually actuated but the device is specifically developed for liquids that can be actuated 
by an EWOD system.

The LCC designed in this paper is an adiabatic component. Adiabatic components are in general more tolerant to fabrication and temperature fluctuations than interference based components [12]. The interference based components, especially when fabricated in silicon, often need to be temperature controlled to maintain a stable operation within the temperature range of the environment where it is used [13]. This involves high energy consumption and an electronics control circuit. Interference-based components can be designed such that they are more tolerant to variations in temperature such as shown in [13]. This, however, increases the complexity of the optical design, and product development time, which should be avoided when possible. Here, we demonstrate that the fabricated adiabatic SiN LCC is inherently tolerant to variations in temperature.

\section{Design of SiN LiQuid controlled COUPLER}

The LCC can be actuated by two liquids with a different refractive index. In Fig. 1 the targeted cross-section of the LCC with SiN waveguides is shown. The oxide cladding on top of the coupler is locally removed by an etching process to expose one of the waveguides to the liquids [3]. As a result, the liquids influence the effective refractive index of the exposed waveguide which allows the switch to operate in bar or cross state. The SiN waveguide height and etch depth are chosen to be $300 \mathrm{~nm}$ and $150 \mathrm{~nm}$, respectively, as this yields a well performing LCC for the chosen EWOD compatible liquid combination as we will show. The thickness of the buried $\mathrm{SiO}_{2}$ cladding is $4.50 \mu \mathrm{m}$ and the thickness of the $\mathrm{SiO}_{2}$ top cladding is $1.00 \mu \mathrm{m}$. The thickness of these claddings is rather large such that the Si substrate and the liquids only above the shielded waveguides have a negligible contribution to the complex effective refractive index of the fundamental quasi-TE mode of the waveguide.

To evaluate the expected performance of the SiN LCCs for different liquid combinations we use a figure-of-merit (FOM) as defined in [3]. For a given combination of liquids, the FOM is defined by finding the set of widths for the input and output waveguides of the coupler which maximizes

$$
\mathrm{FOM}=\min \left(\Delta \beta_{\text {cross, }, \mathrm{n}}, \Delta \beta_{\text {bar,in }}, \Delta \beta_{\text {cross,out }}, \Delta \beta_{\text {bar,out }}\right),
$$

whereby the terms $\Delta \beta$ denote the difference in propagation constants between both input resp. output waveguides, in cross and bar state. An LCC with a higher difference in propagation coefficients at the input and output, and for cross and bar state gives a higher FOM and a better performance in terms of insertion loss and crosstalk for the same length. Fig. 2 shows the FOM over the wavelength range $1260 \mathrm{~nm}$ to $1650 \mathrm{~nm}$ for various refractive index combinations of the 2 LCC actuating liquids. The waveguide cross-section is taken as in Fig. 1 and the cross state is realized with the high index liquid. The graph shows that liquids with refractive indices 1.29 and 1.57 give the highest FOM $\left(0.069 \mu \mathrm{m}^{-1}\right)$. However, we design the LCC for the liquids hydroxypropylene carbonate (HPC, CAS No. 931-40-8) and diphenyl sulfide (DPS, CAS No. 139-66-2) which have a refractive index of 1.44 and 1.60 , respectively, at
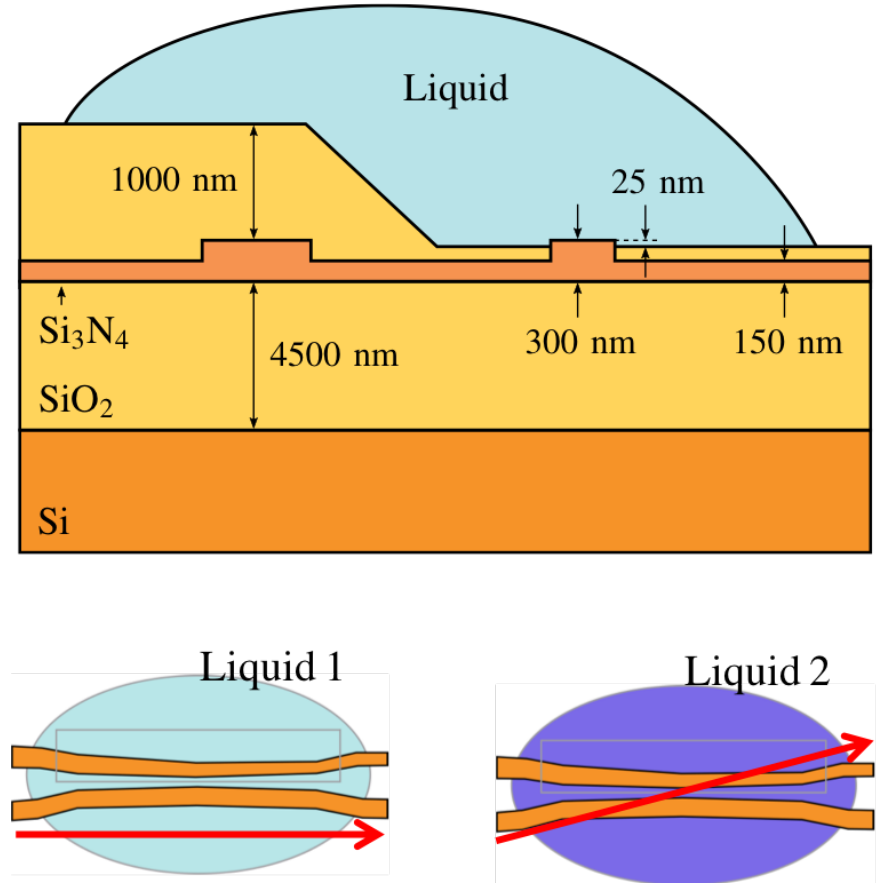

Fig. 1. Cross section and operating principle of the LCC with SiN waveguides. Only one of both waveguides in the adiabatic coupler is in contact with the liquid. With the first liquid on top of the coupler, the switch is in the bar state. If the liquid is interchanged for a second liquid with a different refractive index, the light switches to the cross port

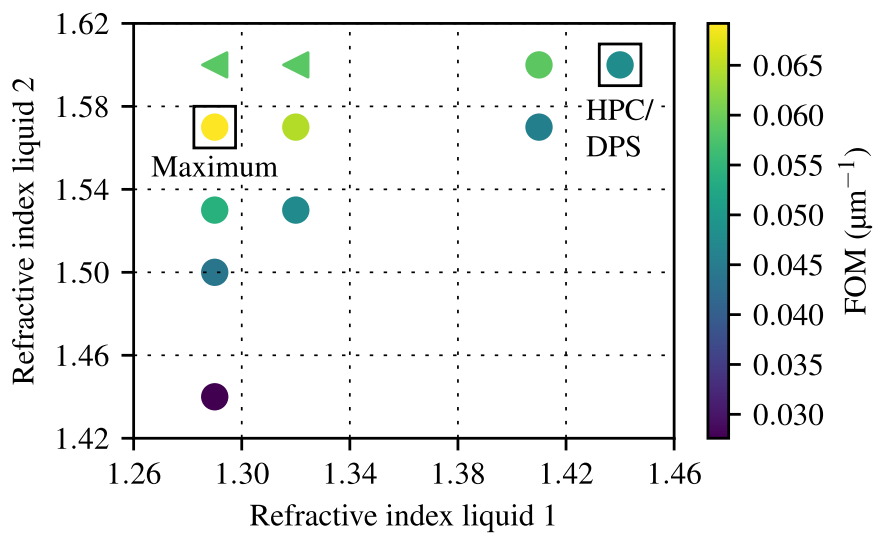

Fig. 2. FOM of SiN LCCs with liquid combinations of various refractive indices over the wavelength range $1260 \mathrm{~nm}$ to $1650 \mathrm{~nm}$.

a wavelength of $1550 \mathrm{~nm}$ and at a temperature of $20^{\circ} \mathrm{C}$. These two liquids are compatible with EWOD systems and can be used to realize a non-volatile switch system such as shown in [11], where we also measured the propagation loss of these liquids to be lower than $4.5 \mathrm{~dB} / \mathrm{cm}$ over the full wavelength range $(1260-1650 \mathrm{~nm})$. The liquids HPC and DPS give an FOM of $0.048 \mu \mathrm{m}^{-1}$.

An LCC where the cross state is realized by the low index liquid is also possible without a reduction in FOM for most of the liquid refractive index combinations shown in Fig. 2. However, for the liquids HPC and DPS a lower FOM is obtained when also fabrication tolerances are taken into consideration. Therefore, the LCC is designed to be in 
cross state for the high index liquid DPS.

For the liquids HPC and DPS the FOM is $0.048 \mu \mathrm{m}^{-1}$ and is higher than that of previously fabricated LCCs with silicon waveguides. The FOM obtained for the Si LCCs described in [3] is $0.02 \mu \mathrm{m}^{-1}$ at $1550 \mathrm{~nm}$ and is $0.01 \mu \mathrm{m}^{-1}$ over the wavelength range $1260 \mathrm{~nm}-1650 \mathrm{~nm}$, for liquids with a refractive index of 1.42 and 1.63. For Si LCCs the FOM is mainly restricted by the difference in the effective refractive index $\left(n_{\mathrm{eff}}\right)$ of the waveguide exposed to the two liquids. For SiN LCCs the difference in $n_{\text {eff }}$ of the waveguide exposed to the two liquids is larger than for $\mathrm{Si}$ and the FOM can now also be restricted by the minimum and maximum allowed waveguide widths. These widths are determined by the single mode operation condition of the separate waveguides (for quasi TE polarization) and the maximum allowed substrate leakage loss. LCCs indicated by triangles in Fig. 2 are restricted by the waveguide width range. LCCs indicated by circles are restricted by the difference in $n_{\text {eff }}$ of the waveguide exposed to the liquids.

The waveguide geometry of the LCC actuated by the liquids HPC and DPS and the simulated transmission spectra are obtained by using a self-consistent orthogonal coupled mode theory such as described in [3] and [14]. Simulated transmission spectra of a $630 \mu \mathrm{m}$ long LCC in bar and cross state are shown in Fig. 3(a) and (b), respectively. The simulated insertion loss in bar state is $0.0 \mathrm{~dB}$ and in cross state it is less than $0.3 \mathrm{~dB}$ over the wavelength range $1260 \mathrm{~nm}$ to 1650 $\mathrm{nm}$. Material and scattering losses are not included in the simulation. The crosstalk in bar state is less than $-52 \mathrm{~dB}$ over the same wavelength range. The crosstalk in cross state is less than $-24 \mathrm{~dB}$ over the wavelength range $1390 \mathrm{~nm}$ to $1650 \mathrm{~nm}$ and is less than $-11 \mathrm{~dB}$ over the wavelength range $1260 \mathrm{~nm}$ to $1650 \mathrm{~nm}$.

The LCC is connected to fiber edge-couplers which interface to optical fibers. The fiber edge-couplers consist of a tapered fully-etched $\mathrm{SiN}$ waveguide with a designed waveguide width of $300 \mathrm{~nm}$ near the chip facet. An index matching fluid can be used to achieve an improved transmission between the fiber edge-coupler and a cleaved optical fiber. When the index matching fluid comes on top of the fiber edge-couplers the shape of the optical mode is more symmetric and thus better matched to the mode profile of the optical fiber which gives an improved transmission. The index matching fluid forms the fiber edge-coupler upper cladding and avoids the need to deposit a thick oxide layer on top of the SiN. When the index matching fluid is also between the chip and optical fiber the reflection is reduced as well.

\section{FABRICATION}

A $300 \mathrm{~nm}$ thick SiN layer is deposited on a $\mathrm{Si}$ wafer with a $4.5 \mu \mathrm{m}$ thick $\mathrm{SiO}_{2}$ layer. A low-pressure chemical vapor deposition (LPCVD) process is used for the SiN deposition. After lithography the SiN layer is partially etched to a depth of $150 \mathrm{~nm}$ using dry etching. This defines the rib waveguides. The fiber edge-couplers contain fully etched waveguides that are realized by a second lithography step. Then, $\mathrm{SiO}_{2}$ is deposited and planarized to a final oxide thickness of $1.0 \mu \mathrm{m}$. The oxide

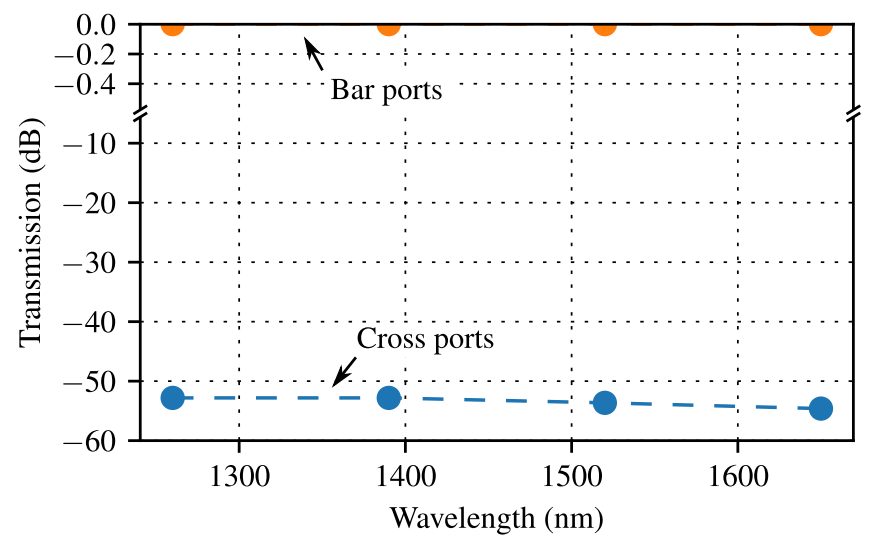

(a)

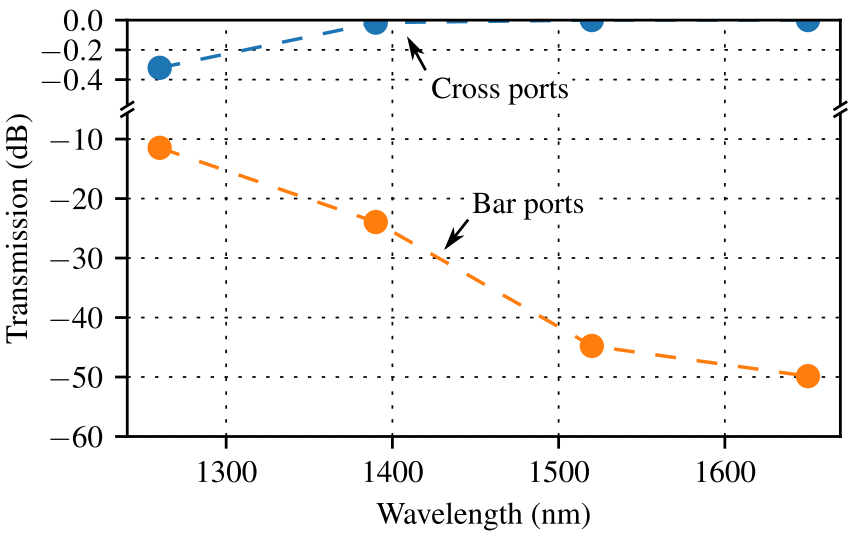

(b)

Fig. 3. Simulated transmission spectra of a SiN LCC in (a) bar state with liquid HPC and (b) in cross state with liquid DPS.



Fig. 4. Microscope image of part of a SiN LCC.

trenches are realized by buffered oxide etching (BOE) in a similar way as described in [3]. A microscope image of one side of the LCC is shown in Fig. 4 and indicates the oxide trench above one of the LCC waveguides.

\section{Characterization AND Discussion}

The LCC is characterized with individual lensed fibers and two tunable Santec laser sources covering the wavelength ranges $1260 \mathrm{~nm}$ to $1360 \mathrm{~nm}$ and $1500 \mathrm{~nm}$ to $1630 \mathrm{~nm}$. The transmission spectra of an LCC in bar and cross state with the liquids HPC and DPS, respectively, are shown in Fig. 5. The transmission is normalized with respect to the average fibre-to-fiber transmission of 2 reference waveguides on the 
same chip. The normalized transmission therefore excludes the transmission of the waveguides and the fiber edge-couplers.

When the LCC is used as a $1 \times 2$ switch, with one input port, and light is launched from input port L1 (orange lines in Fig. 5) then the on-chip loss is less than $0.8 \mathrm{~dB}$ in bar state and less than $1.5 \mathrm{~dB}$ in cross state over the wavelength range $1260 \mathrm{~nm}$ to $1630 \mathrm{~nm}$. The cross state loss is highest at 1630 $\mathrm{nm}$. The crosstalk for this $1 \times 2$ switch is less than $-18 \mathrm{~dB}$ in bar state and less than $-14 \mathrm{~dB}$ in cross state. $1 \times 2$ switches can be used in dilated $2 \times 2$ switches to build large radix switches with reduced crosstalk as will be shown in Section V. The measurement uncertainty of the transmission shown in Fig. 5 is about $0.3 \mathrm{~dB}$ and includes deviations in fiber positioning and the polarization state of the light at the input fiber edgecouplers. The chip is measured at room temperature, $(20.7 \pm$ $0.1)^{\circ} \mathrm{C}$.

In order to use the LCC as a $2 \times 2$ switch, a few improvements are desired. The transmission between ports LO and R0 for the bar state at the lower wavelengths is relatively low $(-4.3 \mathrm{~dB}$ at $1260 \mathrm{~nm})$. Furthermore, the transmission between ports $\mathrm{L} 0$ and $\mathrm{R} 1$ for the cross state at the higher wavelengths is also relatively low $(-3.0 \mathrm{~dB}$ at $1630 \mathrm{~nm})$. A possible reason is that the $600 \mathrm{~nm}$ wide waveguide at port L0 is close to cut-off for the high index liquid DPS. The liquids used to characterize the LCC are the normal formulations of HPC and DPS. When deuterated formulations of HPC and DPS would be used a reduction in the insertion loss can be expected [11]. The deuterated versions have a reduced optical absorption but a similar refractive index. Also, chemical and electrical properties are similar such that they can also be used in an EWOD system. Liquids with reduced absorption are more interesting in LCCs with $\mathrm{SiN}$ waveguides, where the overlap of the light with the liquid is larger, than in LCCs with $\mathrm{Si}$ waveguides.

The LCC is also characterized at the temperatures $51.5^{\circ} \mathrm{C}$ and $73.4^{\circ} \mathrm{C}$. A thermo-electric heater was used and the temperature was measured close to the optical chip. The measured temperature was stable within $\pm 0.2^{\circ} \mathrm{C}$. The transmission of the LCC with input port L1 at different temperatures is shown in Fig. 6(a) and (b) for bar and cross state, respectively. It can be observed that the LCC is tolerant to temperature variations. The excess insertion loss over the temperature range $20.7^{\circ} \mathrm{C}$ to $73.4^{\circ} \mathrm{C}$ is $0.0 \mathrm{~dB}$ and $0.3 \mathrm{~dB}$ for the $\mathrm{LCC}$ in bar and cross state, respectively. The excess crosstalk of the LCC in bar state is $0 \mathrm{~dB}$. The variation in crosstalk of the LCC in cross state due to temperature is larger than in bar state with an excess crosstalk of $6 \mathrm{~dB}$. In cross state the crosstalk reduces at higher wavelengths and increases at lower wavelengths when the temperature increases. Therefore, it can be stated that LCCs, and also adiabatic couplers in general, are less sensitive to variations in temperature when in bar state. Furthermore, the insertion loss and crosstalk of the LCC over the investigated wavelength and temperature range is better in bar state than in cross state.

The LCC is connected to low-loss fiber edge-couplers. The transmission of a fiber edge-coupler is shown in Fig. 7. The measurement uncertainty of the shown transmission is about $0.1 \mathrm{~dB}$. An index matching fluid with a refractive index of 1.42 at a wavelength of $1550 \mathrm{~nm}$ is used. The characterization is done with a high numerical aperture (NA) optical fiber Nufern UHNA4 [15] fusion spliced to a G.652 fiber Corning SMF-28 [16]. The Nufern UHNA4 fiber has a mode field diameter of $4.0 \mu \mathrm{m}$ at a wavelength of $1550 \mathrm{~nm}$ and the Corning SMF-28 fiber has a mode field diameter of $10.4 \mu \mathrm{m}$ at $1550 \mathrm{~nm}$. The splicing loss of about $0.2 \mathrm{~dB}$ is included in the transmission. The fiber-to-fiber transmission of a $1 \times 2$ switch with input port L1 is therefore better than $-5.2 \mathrm{~dB}$ for cross and bar state over the wavelength range $1260 \mathrm{~nm}$ to $1650 \mathrm{~nm}$ at room temperature.

\section{Design of DILATED SWITCh CIRCUIT}

The performance of the demonstrated LCC is better in bar state than in cross state over its wavelength and temperature range of operation. Therefore, in large switch circuits it is advantageous when most LCCs in a light path are in bar state. This can be realized with a cross-bar or a path-independent insertion loss (PILOSS) switch architecture where only one switch element (SE) per light path is in cross state. A SE contains one or more LCCs. When these architectures are used to build large switch circuits the loss contribution of the LCCs in cross state to the total switch loss can then be relatively small. Also the crosstalk of the switch can be improved by using dilated SEs.

A schematic of a $4 \times 4$ dilated cross-bar switch circuit with LCCs is proposed in Fig. 8. The dilated SEs, shown in Fig. 8(b) and (c), consist of 2 LCCS and a waveguide crossover. The 2 LCCs can be positioned next to each other to optimize the footprint of the switch. An EWOD system allows the movement of multiple liquid drops on an optical chip. These liquid drops can be HPC which are surrounded by an ambient liquid such as DPS [11]. When the 2 LCCs are covered by a liquid drop the SE is in bar state, see Fig. 8(b). The crosstalk of this SE in bar state can be two times lower (in logarithmic scale) than that of an elementary LCC. When liquid drops with a circular or slight elliptical shape are used in the EWOD system then the shown dilated SE configuration will not increase the switch footprint compared to SEs with only one LCC due to the large aspect ratio of the SEs. When the SE is in cross state, see Fig. 8(c), the 2 LCCs are covered by the ambient liquid. This deflects the light path from the horizontal to the vertical direction.

It is desired that the crosstalk of the waveguide crossover in the SE is at least 2 times lower in logarithmic scale than the crosstalk of an LCC in bar state. If this is fulfilled then the crosstalk of the dilated $\mathrm{SE}$ in bar state at output port $\mathrm{O}_{1}$, as shown in Fig. 8(b), is about 2 times lower than the crosstalk of an individual LCC. For the demonstrated LCC the crosstalk of the SE is then expected to be less than about $-40 \mathrm{~dB}$ over the investigated wavelength and temperature range. When the $\mathrm{SE}$ is in cross state the crosstalk at output port $\mathrm{O}_{2}$ is identical to that of an LCC. The leaked light is however guided to an unconnected switch port as shown in Fig. 8(a) and (c). The LCC could also be included in a PILOSS switch circuit with an identical SE configuration as shown in Fig. 8. In PILOSS switches the crosstalk of the elementary switches in cross state 
Bar state (HPC)

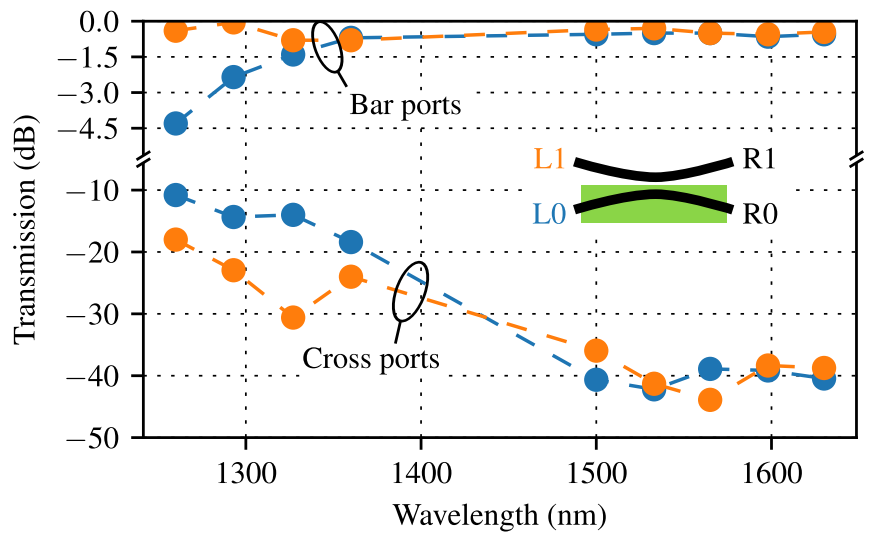

(a)

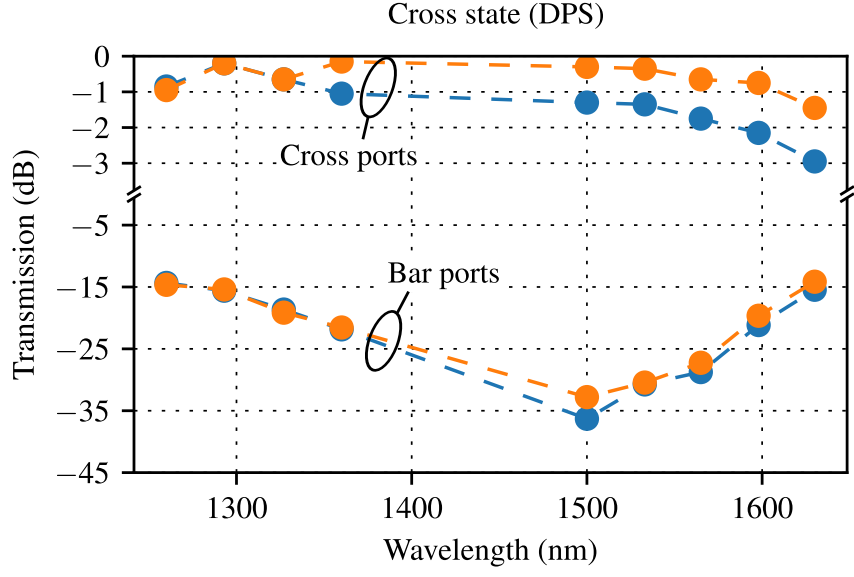

(b)

Fig. 5. Transmission of a SiN LCC in (a) bar state and (b) cross state with liquids HPC and DPS, respectively. The inset shows the port numbers with respect to the waveguide with oxide trench (in green). The transmission spectra with input ports L0 and L1 are shown in blue and orange, respectively. The transmission is normalized with respect to the average fiber-to-fiber transmission of 2 reference waveguides.

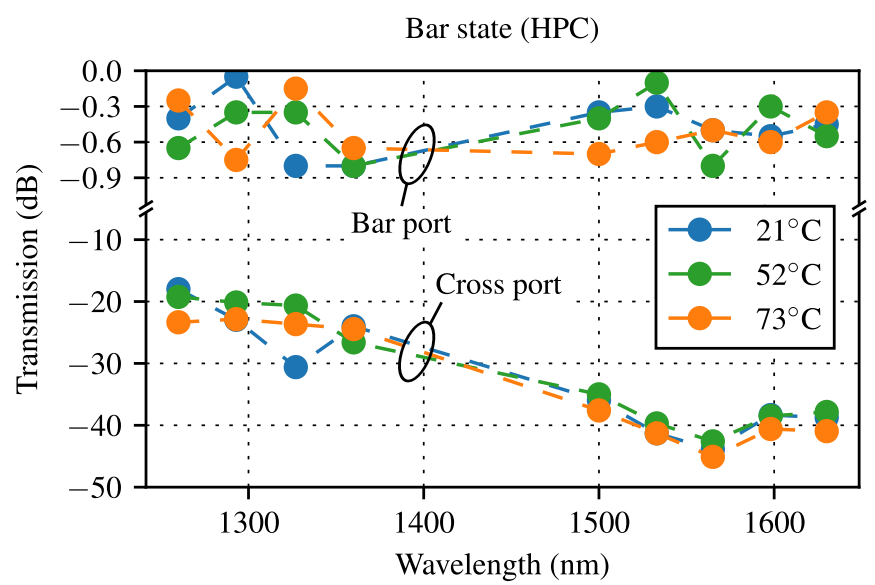

(a)



(b)

Fig. 6. Cross port and bar port transmission of a SiN LCC at different temperatures with input port L1; (a) bar state and (b) cross state.

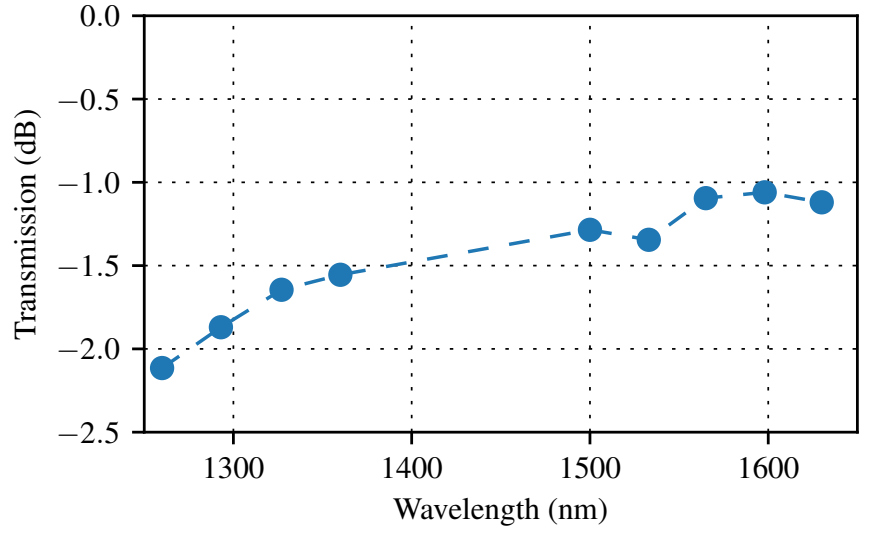

Fig. 7. Loss of a SiN fiber edge-coupler connected to an optical fiber Nufern UHNA4 fusion spliced to a G.652 fiber Corning SMF-28. The loss is determined by halving the measured fiber-to-fiber transmission of a waveguide integrated with 2 edge couplers and includes the $0.2 \mathrm{~dB}$ splicing loss.

can also be guided to unconnected switch ports to achieve a low overall switch crosstalk [17].

For LCCs with a length of $630 \mu \mathrm{m}$, the footprint of a $16 \times 16$ dilated switch circuit that is actuated by an EWOD system is estimated to be as small as $13 \mathrm{~mm} \times 13 \mathrm{~mm}$. A similar size is expected for both a cross-bar and PILOSS switch architecture.

\section{Conclusion}

An ultra-broadband, temperature tolerant, and compact LCC in $\mathrm{SiN}$ has been demonstrated that allows zero energy consumption in a steady switch state. Its operation is demonstrated over the full telecommunication wavelength range from $1260 \mathrm{~nm}$ to $1630 \mathrm{~nm}$ and over the temperature range $21^{\circ} \mathrm{C}$ to $73^{\circ} \mathrm{C}$.

When the LCC is used as $1 \times 2$ switch the $630 \mu \mathrm{m}$ long LCC has an on-chip loss lower than $0.8 \mathrm{~dB}$ in bar state and lower than $1.5 \mathrm{~dB}$ in cross state and has a crosstalk lower than $-18 \mathrm{~dB}$ in bar state and lower than $-14 \mathrm{~dB}$ in cross state over the full wavelength range and at room temperature. 


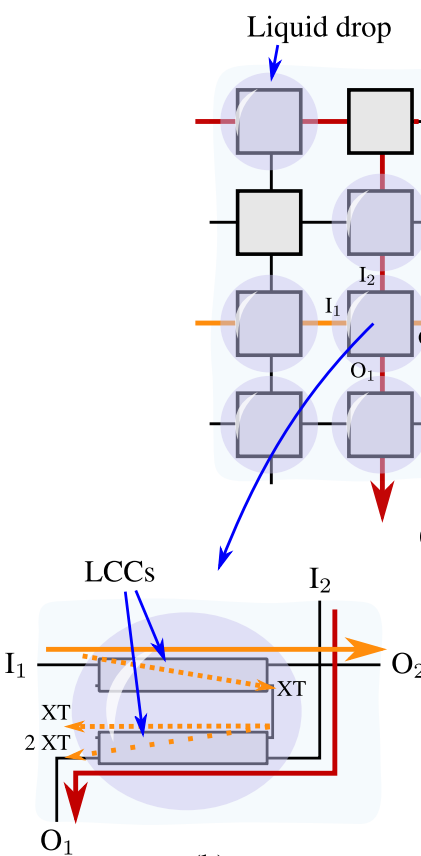

(b)
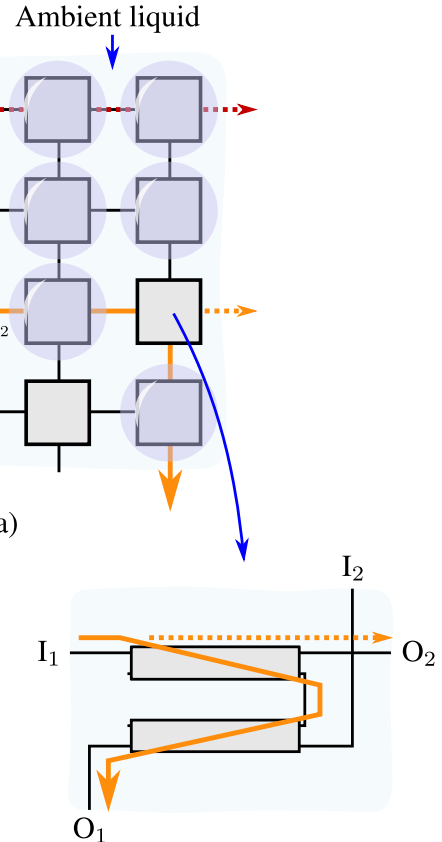

(c)
Fig. 8. Schematic of (a) a cross-bar switch circuit with dilated switch elements in (b) bar state and (c) cross state and with indication of 2 light paths (solid lines) and crosstalk (dotted lines).

While currently the liquids are manually applied, the LCC is designed and characterized with EWOD compatible liquids. EWOD actuation technology allows a non-volatile switch operation, meaning the switch state can be retained without energy consumption [11]. An important aspect in assuring reliable EWOD operation is avoiding residues after switching the liquids. This requires a suitable dewetting coating, which is sufficiently thin $(; 10 \mathrm{~nm})$ to not affect the optical characteristics of the device. Recent experiments using a silane monolayer showed promising results [18].

Furthermore, it is demonstrated that the LCC is tolerant to variations in temperature. The excess insertion loss over the temperature range $20.7^{\circ} \mathrm{C}$ to $73.4^{\circ} \mathrm{C}$ is $0.0 \mathrm{~dB}$ for the bar state and $0.3 \mathrm{~dB}$ for the cross state. A passive LCC with a high tolerance to temperature enables a stable switch operation in environments with large variations in temperature without additional energy consumption in the form of heating or cooling. Although the LCC was not characterized at lower temperatures, we expect that it is feasible to implement these switches in datacenters and central offices without temperature control where the temperature specification is between $-5^{\circ} \mathrm{C}$ and $65^{\circ} \mathrm{C}$. The current switch is designed for a single polarisation (quasi TE). To allow polarisation independent operation a polarisation diversity scheme should be implemented. In principle this should be possible without increasing the chip size, as the two LCCs addressing orthogonal polarisations can be driven by a single EWOD cell.

The SiN LCCs are ideally suited in applications where the state of the switches are sporadically changed. Due to its zeroenergy consumption after switching and because no thermal control would be required, large amounts of energy can be saved. On top of that, its small footprint enables the realization of dense switch circuits. One example of use is in a switch that manages the reconfiguration of optical fiber communication networks.

\section{ACKNOWLEDGMENT}

The authors would like to thank S. Severi and B. Du Bois for the fruitful discussion about wafer processing.

\section{REFERENCES}

[1] V. Intaraprasonk and S. Fan, "Nonvolatile bistable all-optical switch from mechanical buckling," Apll. Phys. Lett., vol. 98, no. 24, p. 241 104, Jun. 2011.

[2] S. Abe and K. Hane, "Variable-gap silicon photonic waveguide coupler switch with a nanolatch mechanism," IEEE Photon. Technol. Lett., vol. 25, no. 7, pp. 675-677, Apr. 2013.

[3] H. D'heer et al., "Nonvolatile liquid controlled adiabatic silicon photonics switch," J. Lightw. Technol., vol. 35, no. 14, pp. 2948-2954, Jul. 2017.

[4] T. J. Seok, N. Quack, S. Han, R. S. Muller, and M. C. Wu, "Large-scale broadband digital silicon photonic switches with vertical adiabatic couplers," Optica, vol. 3, no. 1, pp. 64-70, Jan. 2016.

[5] J. V. Campenhout, W. M. J. Green, S. Assefa, and Y. A. Vlasov, "Lowpower, $2 \times 2$ silicon electro-optic switch with $110-\mathrm{nm}$ bandwidth for broadband reconfigurable optical networks," Opt. Express, vol. 17, no. 26, pp. $24020-24029$, Dec. 2009.

[6] S. Chen, Y. Shi, S. He, and D. Dai, "Low-loss and broadband $2 \times 2$ silicon thermo-optic mach-zehnder switch with bent directional couplers," Opt. Lett., vol. 41, no. 4, pp. 836-839, Feb. 2016.

[7] H. D'heer et al., "A $16 \times 16$ non-volatile silicon photonic switch circuit," IEEE Photonics Technology Letters, vol. 30, no. 13, 1258-1261, 2018.

[8] J. E. Fouquet, "Compact optical cross-connect switch based on total internal reflection in a fluid-containing planar lightwave circuit," in Optical Fiber Communication Conference. Technical Digest Postconference Edition. Trends in Optics and Photonics Vol.37 (IEEE Cat. No. 00CH37079), vol. 1, 2000, 204-206 vol.1.

[9] D. Psaltis, S. R. Quake, and C. Yang, "Developing optofluidic technology through the fusion of microfluidics and optics," Nature, vol. 442 , $381 \mathrm{EP}-$, Jul. 2006.

[10] C. L. Arce, D. Witters, R. Puers, J. Lammertyn, and P. Bienstman, "Silicon photonic sensors incorporated in a digital microfluidic system," English, Analytical and bioanalytical chemistry, vol. 404, no. 10, 2887-2894, 2012.

[11] S. Günther et al., "EWOD system designed for optical switching," in Proc. IEEE 30th Int. Conf. Micro Electro Mech. Syst., 2017, pp. 13291332.

[12] M. G. F. Wilson and G. A. Teh, "Improved tolerance in optical directional couplers," Electron. Lett., vol. 9, no. 19, pp. 453-455, Sep. 1973.

[13] S. Dwivedi, H. D'heer, and W. Bogaerts, "A compact all-silicon temperature insensitive filter for WDM and bio-sensing applications," IEEE Photon. Technol. Lett., vol. 25, no. 22, pp. 2167-2170, Nov. 2013.

[14] H. Ishikawa, "Fully adiabatic design of waveguide branches," J. Lightw. Technol., vol. 25, no. 7, pp. 1832-1840, Jun. 2007.

[15] Nufern, Inc. (2017), [Online]. Available: www.nufern.com.

[16] Corning, Inc. (2017), [Online]. Available: www.corning.com.

[17] K. Suzuki et al., "Broadband silicon photonics $8 \times 8$ switch based on double-Mach-Zehnder element switches," Opt. Express, vol. 25, no. 7 , pp. 7538-7546, Apr. 2017.

[18] S. Günther et al., "Electrowetting controlled non-volatile integrated optical switch," Accepted for the European Conference on Optical Communnications, ECOC 2018, 2018. 